\title{
EFECTO DEL CAMBIO CLIMATICO SOBRE EL RENDIMIENTO DE LOS CULTIVOS DE LA REGIÓN LIMA 2007-2015
}

\author{
EFFECT OF CLIMATE CHANGE ON THE YIELD OF CROPS IN THE LIMA REGION 2007-2015
}

\author{
Lucas Saul Leyva Chinchay ${ }^{1}$, Sergio Eduardo Contreras Liza ${ }^{2}$
}

\begin{abstract}
RESUMEN
Objetivo: Presentar evidencia de los efectos potenciales del cambio climático sobre el sector agrario en la Región Lima en el período 2007-2015. Métodos: Fue una investigación no experimental y descriptiva, con acopio de información de fuentes oficiales. Se tomó la data del Compendio Estadístico, elaborado por el Ministerio de Agricultura, SENAMHI, INEI, CENAGRO, entre otras fuentes de información. Resultados: Los cultivos más afectados fueron, caña de azúcar, papa, paprika, cítricos y palta y los que aumentaron sus rendimientos fueron algodón, camote, yuca, frijol, mango, maracuyá, papaya y tangelo. Las áreas con bosques de protección se han reducido en 74,5\% entre 1994 y 2015.Conclusiones: Los resultados indican que la variabilidad en los rendimientos de los cultivos puede deberse en parte a las alteraciones climáticas, que producen cambios significativos en la fisiología de las plantas, debiendo adoptarse medidas para reducir el impacto ambiental, sobre todo con una política de reforestación a nivel regional.
\end{abstract}

Palabras clave: Variabilidad climática; Impacto ambiental; Reforestación; Región Lima.

\begin{abstract}
Objective: To present evidence of the potential effects of climate change on the agricultural sector in the Lima Region in the 2007-2015 period. Methods: It was a non-experimental and descriptive investigation, with information gathering from official sources. The data was taken from the Statistical Compendium, prepared by the Ministry of Agriculture, SENAHMI, INEI, CENAGRO, among other sources of information. Results: The crops most affected were sugarcane, potatoes, paprika, citrus fruits and avocado and those that increased their yields were cotton, sweet potato, cassava, beans, mango, passion fruit, papaya and tangelo. The areas with protection forests have been reduced by $74.5 \%$ between 1994 and 2015 . Conclusions: The results indicate that the variability in crop yields may be due in part to climatic alterations, which produce significant changes in the physiology of the plants; strategies must be taken in account to reduce the environmental impact, especially with a reforestation policy at regional level.
\end{abstract}

Key words: Climatic variability; Environmental impact; Reforestation; Lima region.

\footnotetext{
${ }^{1}$ Director de Información Agraria de la región Lima. Email: saul_leyva@hotmail.com

${ }^{2}$ Docente de la Universidad Nacional José Faustino Sánchez Carrión de Lima (Lima - Perú).
} 


\section{INTRODUCCIÓN}

De acuerdo al INEI (2015) la población de la región Lima Provincias fue de 923682 habitantes con una densidad poblacional de 28,75 habitantes por $\mathrm{km}^{2} \mathrm{y}$ una extensión de $32435 \mathrm{~km}^{2}$, que representa el 2,5\% del territorio nacional. El cambio climático implica un reto muy importante en nuestra región ya que se tiene aún pendiente la reducción de la pobreza en la que vive una parte importante de la población rural. A pesar de que se necesita mayor investigación para entender los determinantes de la adaptación de los cultivos, es claro que las intervenciones para promover y facilitar la adaptación al cambio climático no deben estar desligadas del desarrollo rural, la disminución de la pobreza y el manejo de los recursos naturales. Es decir, la adaptación al cambio climático no debe verse como un tema aislado sino como parte de una estrategia de desarrollo.

En el Perú y en algunas regiones, se están dedicando esfuerzos importantes a desarrollar políticas públicas para promover la adaptación al cambio climático. Sin embargo, es importante que se profundice el entendimiento sobre la forma en la que los agricultores perciben los riesgos climáticos en sus provincias, distritos. Una vez que se cuente con esa información, podrán diseñarse políticas de adaptación que resulten relevantes localmente.

El sector agrícola juega un rol importante en la seguridad alimentaria. Ningún país ha logrado sostener un proceso de crecimiento económico rápido sin antes resolver el problema de seguridad alimentaria. Esto es necesario para el crecimiento, puesto que el acceso inadecuado e irregular a los alimentos limita la productividad y reduce la inversión en capital humano (Ortiz, 2012).

A nivel macroeconómico, las crisis alimentarias recurrentes afectan la estabilidad política y económica lo que a su vez reduce la eficiencia de la inversión La agricultura afectada por variables no controladas puede tener efectos macroeconómicos negativos, principalmente si existe demasiada volatilidad en los precios de los alimentos (LopezFeldman, 2015).

De acuerdo con Ortiz (2012), estos efectos son:

i) disminución en la inversión debido a mayores niveles de incertidumbre;

ii) los precios de los alimentos pueden tener efectos desestabilizadores en los precios de otros bienes y servicios; iii) si los precios de los bienes agrícolas aumentan el nivel de riesgo en la economía, entonces la inversión se aleja de las actividades productivas y se mueve hacia las actividades especulativas.

Brown et al., (2015) mostrando los cambios de temperatura desde 1880 hasta 2015 , advierte que el año 2015 fue el año más caluroso. Este incremento de la temperatura coincide con el desarrollo de la Revolución Industrial, momento a partir del cual la acción del hombre se hizo más intensa sobre la naturaleza. Ante ello, se viene alertando de que si el desarrollo mundial, el crecimiento demográfico y el consumo energético basado en los combustibles fósiles, siguen aumentando al ritmo actual, antes del año 2050 las concentraciones de dióxido de carbono se habrán duplicado con respecto a las que había antes de la Revolución Industrial. Si la concentración de $\mathrm{CO}_{2}$ en la atmósfera continua por encima de las 350 partes por millón (ppm), puede haber consecuencias en los ecosistemas.

El tercer informe del IPCC (2001) resalta algunos de los impactos del Cambio Climático sobre la pobreza a nivel global:

I. Disminución en el acceso al agua, que afectará la productividad de las tierras agrícolas y generará la aparición de nuevas plagas. Se espera una caída en la productividad de las tierras hasta en un $30 \%$ sobre el siglo XXI.

II. Masivos desplazamientos de poblaciones afectadas de las zonas altas hacia la costa, la baja producción y el déficit de agua de las zonas bajas orientara hacia las grandes ciudades debido a inundaciones y salinización de áreas fértiles, dejando sin lugar para vivir a miles de personas

III. Aumento en la frecuencia y severidad de fenómenos climáticos extremos como El Niño, huracanes, sequías e inundaciones

Uribe - Botero (2015) menciona que las principales causas del incremento de la temperatura son: la deforestación, al crecer los bosques absorben el carbono de la atmosfera y los almacenan en su biomasa y en el suelo; el $\mathrm{N}_{2} \mathrm{O}$ procedente de los fertilizantes nitrogenados, la agricultura intensiva, los cultivos intensivos contaminan la atmosfera por la emisión y quema de los combustibles; liberación del carbono del suelo a la atmosfera, incrementando la descomposición y mineralización de la materia orgánica, reduciendo la materia orgánica por oxidación y provocando la erosión eólica e hídrica del suelo; el metano procedente de la fermentación 
intestinal de los animales rumiantes.

\section{MATERIALES Y MÉTODOS}

El diseño de investigación fue no experimental y descriptivo. Las técnicas de recolección de información fueron: recopilación de fuentes de informaciones primarias y secundarias y diagnóstico exploratorio. Para la evaluación de este trabajo se tomó como referencia el compendio estadístico, elaborado por el ministerio de agricultura, y los informes anuales de la Dirección de Información del gobierno Regional; con dichos documentos se elaboraron las tablas resumen con el efecto del incremento de la temperatura sobre el rendimiento de los cultivos en la región Lima durante el periodo 2007-2015.Para corregir algunos efectos del tipo de suelos y de prácticas de cultivos, se consideró que los rendimientos menores de $500 \mathrm{~kg} / \mathrm{ha}$, con respecto al año anterior no eran significativos y cultivos con una variación mayor, como significativos para el agricultor.

Se tomó la información de los datos meteorológicos de temperatura (SENAMHI) y su tendencia promedio en la región (2007-2015. Finalmente, se evaluó las pérdidas de los bosques y montes en la región Lima, en forma comparativa entre 1994 y el 2015, usando datos del CENAGRO e INEI. Los datos fueron procesados con el programa estadístico Infostat.

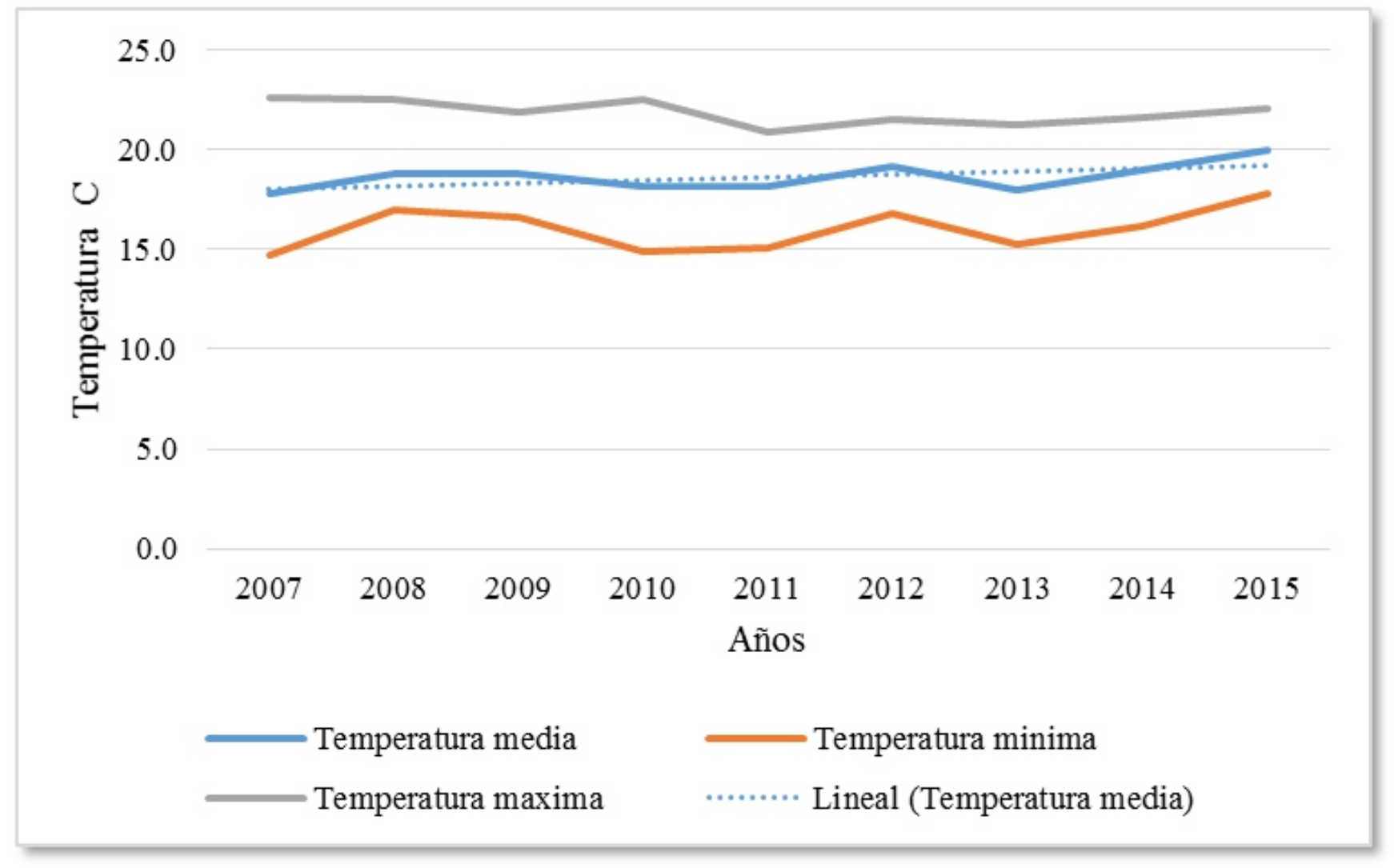

Figura 1. Región Lima: Temperaturas máximas, mínimas y medias. Elaboración propia basada en registros de SENAMHI (http://www.senamhi.gob.pe/? $p=$ descarga-datos-hidrometeorologicos)

\section{RESULTADOS}

La tabla 1 muestra los cultivos que incrementaron sus rendimientos medios entre 2007-2015, resaltando principalmente especies anuales como camote, col o repollo, esparrago, tomate, yuca y zapallo y dentro de los frutales limón sutil, pepino, mango, maracuyá, papaya, tangelo y vid, siendo los que incrementaron sus rendimientos en relación a años anteriores. 
Tabla 1. Cultivos que fueron favorecidos por el cambio climático y que incrementaron sus rendimientos (t/ha.)

\begin{tabular}{|c|c|c|c|c|c|c|c|c|c|c|}
\hline Producto & 2007 & 2008 & 2009 & 2010 & 2011 & 2012 & 2013 & 2014 & 2015 & $\begin{array}{c}\text { Variación } \\
\%\end{array}$ \\
\hline Ajo & 7,164 & 6,930 & 6,691 & 7,098 & 7,082 & 6,952 & 7,183 & 6,551 & 6,785 & $+3,57$ \\
\hline Algodón & 2,949 & 2,867 & 2,966 & 2,976 & 2,815 & 3,061 & 2,956 & 2,681 & 3,156 & $+17,72$ \\
\hline Caigua & 9,129 & 7,274 & 7,477 & 6,947 & 6,188 & 5,563 & 5,035 & 4,267 & 5,238 & $+22,76$ \\
\hline Camote & 19,880 & 21,54 & 20,22 & 19,83 & 20,47 & 20,19 & 21,35 & 20,69 & 21,51 & $+3,99$ \\
\hline Cebada grano & 1,589 & 1,491 & 1,652 & 1,603 & 1,681 & 1,750 & 1,502 & 1,850 & 2,069 & $+11,84$ \\
\hline Ciruela & 14,54 & 13,96 & 14,93 & 14,72 & 14,75 & 13,11 & 15,480 & 10,61 & 11,41 & $+7,61$ \\
\hline Col o repollo & 13,48 & 14,85 & 12,95 & 11,98 & 10,78 & 10,54 & 10,510 & 10,86 & 12,19 & $+12,23$ \\
\hline Espárrago & 8,724 & 9,011 & 7,605 & 7,971 & 9,597 & 9,415 & 8,845 & 9,070 & 9,896 & $+9,11$ \\
\hline Frijol vainita & 7,159 & 7,023 & 7,610 & 7,159 & 7,249 & 7,421 & 7,646 & 7,179 & 7,554 & $+5,22$ \\
\hline Lechuga & 5,740 & 6,745 & 7,349 & 7,043 & 6,687 & 6,025 & 6,423 & 6,859 & 7,081 & $+3,24$ \\
\hline Lima & 7,000 & 7,000 & 7,077 & 11,54 & 9,067 & 8,800 & 6,923 & 6,900 & 10,65 & $+54,30$ \\
\hline Limón sutil & 10,82 & 8,964 & 6,273 & 5,100 & 6,667 & 7,857 & 7,143 & 8,167 & 10,17 & $+24,49$ \\
\hline Mango & 9,607 & 9,549 & 7,848 & 7,831 & 7,393 & 7,305 & 12,01 & 11,85 & 13,43 & $+13,36$ \\
\hline Maní & 3,235 & 3,830 & 3,558 & 3,362 & 3,259 & 3,789 & 3,792 & 3,162 & 3,573 & $+13,00$ \\
\hline Maracuyá & 16,6 & 14,08 & 13,68 & 14,93 & 14,37 & 14,76 & 13,05 & 11,85 & 14,54 & $+22,71$ \\
\hline Melón & 22,93 & 21,56 & 21,59 & 20,63 & 20,56 & 19,89 & 20,08 & 19 & 19,44 & $+2,36$ \\
\hline Nabo & 15,000 & 11,889 & 18,000 & 9,500 & 7,615 & 9,692 & 9,250 & 7,235 & 10,000 & $+38,22$ \\
\hline Papaya & 10,32 & 7,800 & 10,800 & 10,85 & 9,000 & 9,308 & 9,417 & 10,32 & 12,310 & $+19,24$ \\
\hline Pepino & 28,44 & 26,55 & 26,5 & 24,36 & 22,56 & 19,95 & 24,41 & 27,07 & 30,090 & 11,14 \\
\hline Tangelo & 29,97 & 29,54 & 28,6 & 38,85 & 37,47 & 42,36 & 49,850 & 39,12 & 42,430 & $+8,47$ \\
\hline Uva & 15,58 & 16,56 & 15,86 & 16,15 & 15,58 & 16,120 & 17,19 & 17,95 & 18,9 & $+5,29$ \\
\hline Yuca & 30,77 & 32,52 & 33,65 & 34,86 & 33,49 & 33,98 & 34,37 & 35,25 & 37,47 & $+6,31$ \\
\hline
\end{tabular}

En la Tabla 2. Se observan los cultivos que fueron afectados por el cambio climático y que redujeron sus rendimientos posiblemente por altas temperaturas ambientales en el periodo 2007-2015. 
Tabla 2. Cultivos que fueron afectados por el cambio climático y que redujeron sus rendimientos (t/ha)

\begin{tabular}{lcccccccccc}
\multicolumn{1}{c}{ Producto } & $\mathbf{2 0 0 7}$ & $\mathbf{2 0 0 8}$ & $\mathbf{2 0 0 9}$ & $\mathbf{2 0 1 0}$ & $\mathbf{2 0 1 1}$ & $\mathbf{2 0 1 2}$ & $\mathbf{2 0 1 3}$ & $\mathbf{2 0 1 4}$ & $\mathbf{2 0 1 5}$ & $\begin{array}{c}\text { Variación } \\
\mathbf{\%}\end{array}$ \\
\hline Aceituna & 5,713 & 6,329 & 6,470 & 6,109 & 8,41 & 8,138 & 9,152 & 9,024 & 7,362 & $-18,42$ \\
Albahaca & 9,750 & 9,727 & 9,789 & 10,10 & 9,50 & 11,29 & 9,000 & 8,733 & 6,600 & $-24,42$ \\
alcachofa & 13.48 & 13.86 & 16.86 & 16.92 & 15.96 & 15.88 & 15.63 & 12.73 & 11.7 & -8.14 \\
Arándano & & & & & & 7,000 & 11,00 & 7,583 & 3,727 & $-50,85$ \\
Caña de azúcar & 44,36 & 29,400 & 95,900 & 106,1 & 72,7 & 84,75 & 26,33 & 42,57 & 35,33 & $-17,00$ \\
Cebolla & 30,18 & 31,492 & 28,32 & 26,33 & 29,6 & 27,27 & 30,89 & 31,54 & 30,08 & $-4,61$ \\
Guayaba & 9,000 & 8,333 & 8,538 & 8,538 & 6,800 & 6,500 & 7,000 & 8,000 & 4,333 & $-45,84$ \\
Lúcuma & 10,17 & 9,844 & 10,760 & 10,53 & 9,39 & 10,77 & 11,01 & 12,460 & 10,84 & $-12,99$ \\
Maíz choclo & 13,7 & 15,150 & 16,62 & 18,03 & 18 & 17,06 & 16,17 & 17,24 & 14,91 & $-13,55$ \\
Mandarina & 31,25 & 30,545 & 23,55 & 33,81 & 32,6 & 37,42 & 38,79 & 40,95 & 36,23 & $-11,53$ \\
Naranja & 24,71 & 25,938 & 26,81 & 27,88 & 34,6 & 35,27 & 35 & 40,27 & 35,05 & $-12,97$ \\
Papa & 23,95 & 23,812 & 20,82 & 25,54 & 23,9 & 21,36 & 23,9 & 23,74 & 18,12 & $-23,68$ \\
Palta & 10,96 & 11,16 & 11,49 & 11,72 & 10,71 & 12,92 & 13,02 & 12,85 & 12,11 & $-5,80$ \\
Páprika & 3,685 & 5,112 & 3,881 & 4,832 & 5,91 & 5,376 & 5,683 & 6,262 & 5,521 & $-11,83$ \\
Toronja & 17,000 & 26,500 & 11,88 & 16,88 & 17,9 & 17,44 & 28,22 & 22,00 & 19,200 & $-12,73$ \\
\hline
\end{tabular}

En la tabla 3 observamos que se redujeron 34,184.82 hectáreas de montes que captaban el dióxido de carbono, sin contar las pérdidas de las riberas de los ríos que actuaban mejorando la infiltración del agua para ser aprovechadas en las zonas medias y bajas.

Tabla 3. Reducción de los bosques (has) por deforestación en la región Lima.

\begin{tabular}{lcc}
\hline Agencias & $\mathbf{1 9 9 4} *$ & $\mathbf{2 0 1 5 * *}$ \\
\hline Barranca & 7589,35 & 567,0 \\
Cajatambo & 25,18 & 33,09 \\
Gorgor & 104,89 & 279,65 \\
\hline Huacho & 1898,27 & 141,66 \\
Oyon & 800,82 & 3871,04 \\
Sayan & 1293,68 & 1626,5 \\
\hline Huaral & 6543,94 & 80,46 \\
Acos & 4042,96 & 42,95 \\
\hline Canta & 320,08 & 204,06 \\
Santa Rosa & 80,98 & 6,78 \\
\hline Santa Eulalia & 2798,19 & 39,06 \\
Matucana & 144,84 & 174,77 \\
Huarochirí & 7572,85 & 36,05 \\
Langa & 6410,82 & 133,5 \\
\hline Mala & 87,04 & 73,63 \\
Quinches & 1002,86 & 180,25 \\
\hline Cañete & 134,34 & 364,68 \\
Yauyos & 826,20 & 60,92 \\
Catahuasi & 3265,25 & 3237,67 \\
\hline Total & $45,338,54$ & $11,153,72$ \\
\hline & & \\
\hline
\end{tabular}

*Fuente: Cenagro 1994

** Fuente: Dirección Regional Agricultura Lima Provincias 


\section{DISCUSIÓN}

La temperatura actúa sobre los órganos de las plantas como un factor iniciador y regulador de los procesos del metabolismo, crecimiento y desarrollo. En algunos casos los órganos pueden morir incluso la propia planta, cuando la temperatura sobresale de los rangos determinados. La temperatura influye en la difusión de los gases y líquidos en la planta, en la solubilidad de los iones y la viscosidad del agua, afectando la velocidad de transporte y la transpiración, la temperatura aérea es muy importante como también la temperatura radicular.

Fernández (2006) encontró que las plantas de origen de zonas templadas el crecimiento por alargamiento y la división celular de las partes aéreas se inicia por debajo de los $10^{\circ} \mathrm{C}$ y en las plantas de origen tropical ocurre entre $12-15^{\circ} \mathrm{C}$, necesitando de mayor calor, para la división celular y en la mayoría de las plantas herbáceas la temperatura optima se encuentra entre $\operatorname{los} 30^{\circ} \mathrm{C}$. La tasa de crecimiento longitudinal de las células es más alta en plantas tropicales y subtropicales se encuentra entre $30-40{ }^{\circ} \mathrm{C}$ y en las demás plantas esta entre $15-30{ }^{\circ} \mathrm{C}$ (Manso et al., 2008). En el caso del camote, Fisher, Carbajal \& Torres (1995) mencionan que las temperaturas del suelo alrededor del suelo de $24{ }^{\circ} \mathrm{C}$ son adecuados para la formación del tubérculo, mientras que $22{ }^{\circ} \mathrm{C}$ son ideales para su desarrollo posterior, pero cuidado la temperatura de $30^{\circ} \mathrm{C}$ en el suelo fomentan la producción de citoquinina en las puntas radicales y su transporte al sistema aéreo.

Melgarejo (2016) reportó que en Cañete la temperatura varia de 19,7 a $22,8{ }^{\circ} \mathrm{C}$, y en los primeros meses del año 2016 se elevó a $29,3{ }^{\circ} \mathrm{C}$. En el caso de la papa las temperaturas altas fomentan el crecimiento del follaje, reducen el crecimiento de los tubérculos, al inicio su crecimiento es rápido $\mathrm{y}$ en mayor número con temperaturas del suelo relativamente bajas $10-15^{\circ} \mathrm{C}$, mientras entre $15^{\circ}$ $20^{\circ}$, se forman menos tubérculos, creciendo más rápido.
La menor producción de tubérculos y el follaje alto son causados por las temperaturas altas del suelo; ello se atribuye a una mayor síntesis de gibelinas que produce un enviciamiento, sombreamiento que inhiben o por lo menos la iniciación de estos órganos subterráneos (Midmore, 1988)

Es necesario recuperar las áreas de protección por bosques ya que se perdió hasta la fecha, el 74.5\% del área de conservación con especies perennes. Leyva-Chinchay (2015) en un estudio de valoración de la estructura y dinámica de los bosques fluviales en el rio Huaura encontró que la calidad de los bosques afectados en 55\%, requiriéndose su repoblación. La importancia de recuperar las zonas ribereñas es vital porque los bosques ribereños son sumideros de $\mathrm{CO}_{2}$ contribuyendo a reducir los gases de efecto invernadero.

\section{CONCLUSIONES}

Los cultivos en la región Lima tuvieron dos formas principales de comportamiento en cuanto a rendimiento: los que mejoraron por efecto de variabilidad climática y los cultivos que fueron afectados durante el periodo 2007-20015.

Los cultivos más afectados en su rendimiento fueron la caña de azúcar $(-17 \%)$, papa $(-23 \%)$, páprika $(-11 \%)$, choclo $(-13 \%)$, lúcuma $(-13 \%)$, palta $(-5 \%)$.

Los cultivos que mejoraron su rendimiento fueron: algodón $(+17 \%)$, yuca $(+6 \%)$, frijol $(+5 \%)$, camote $(+4 \%)$, espárrago $(+9 \%)$, limón $(+24 \%)$, maracuyá $(+22 \%)$, papaya $(+19 \%)$, mango $(+13 \%)$, tangelo $(+8 \%)$, uva de mesa $(+5 \%)$.

Se requiere iniciar una política de forestación en las partes altas y en las riberas de los ríos considerando las áreas de montes fueron reducidas, para incrementar el flujo de aguas subterráneas y recuperar la funcionalidad de los suelos. 


\section{REFERENCIA BIBLIOGRÁFICA}

Brown, D., Cabbage, M., McCarthy, L., \& Norton, K. (2016). NASA, NOAA analyses reveal record-shattering global warm temperatures in 2015. Press release (available on the internet at http://www. nasa. gov/press-release/nasanoaaanalyses-reveal-record-shattering-globalwarm-temperatures-in-2015). Recuperado 23 agosto 2017.

Fernández G (2006) Crecimiento y Temperatura. Ed Universidad de la Serena, La serena, Chile 20(20): 12-17

Fisher G, Carbajal F,Torres J. (1995) Efectos de la temperatura del suelo sobre la planta.Rev Comalfi 24(3), 78-92

IPCC. (2001). Informe especial del IPCC: Uso de la tierra, cambio de uso de la tierra y silvicultura. Informe especial del Grupo de Trabajo III del Grupo Intergubernamental de expertos en cambio climático.

Leyva-Chinchay S. (2015) Buenas prácticas agrícolas y el uso de los fertilizantes nitrogenados en la minimización de la emisión de gases de efecto de invernadero. Rev. Ingetecno. 4(1), 8
López-Feldman, A. (2015) Cambio climático las actividades agropecuarias en América Latina. CEPAL. Naciones Unidas, Santiago de Chile. $76 \mathrm{p}$.

Melgarejo M. (2016) Informe efecto del Niño. Agencia Agraria Cañete Informe Interno.

Manso JM, de Azagra Paredes, A M. (2008). Restauración de los suelos y de la vegetación en la lucha contra la desertificación. Cuadernos de la Sociedad Española de Ciencias Forestales, (25):309-313

Midmore D (1988) Fisiología de la planta de papa en climas calidos.Guia de invest. CIP 24. Centro Internacional de la papa. Lima Perú

Ortiz R. (2012) El cambio climático y la producción agrícola. Notas técnicas 383 BANCO Interamericano de desarrollo.

Uribe-Botero E (2015) Cambio climático y sus efectos en la biodiversidad en américa Latina. CEPAL. Naciones Unidas. Santiago de Chile $55 \mathrm{p}$. 